\title{
A rapid technique for normalizing scores'
}

WALTER TAUSS

UNIVERSITY OF WESTERN AUSTRALIA

By using a new form of statistical table, frequency distributions can be readily transformed into approximately normal distributions without the need for elaborate computations or programs. The technique is illustrated by means of a worked example.

Many statistical procedures are based on the assumption of normally distributed data (for example, CR tests, correlation coefficients, or analysis of variance). Unfortunately, real data do not always meet these requirements, so that the researcher must resort to transformations employing square roots, logarithms, trigonometric ratios, or a combination of more than one of these. Even then, the transformed data may not always conform to the desired pattern of distribution, although valuable computer time has been spent in an endeavor to make them do so.

In sociological or psychological research one often deals with quasi-scales, and the assumption of an underlying normally distributed continuum is logically no less defensible than is the common practice of adding point scores to obtain a series of numerical values which are then treated as if they formed real scales. Fisher (1966, p. 4) presents diagrams illustrating percentage areas into which the standard normal distribution may be divided. In a subsequent paper, Fisher (1967) discusses how these tables may be used to test the distribution of actual data for normality.

It is suggested that the same tables may be used to effect a rapid transformation with the minimum of computational tedium. If the total number of observations is reasonably well spread over a wide numerical range and is relatively large, the distribution of transformed data would then in all cases closely approximate a normal distribution. This technique can deal with relatively large skews, provided the frequencies falling into classes at the extremes of the range are small.

Table 1

\begin{tabular}{cc} 
Interval & $\begin{array}{c}\text { Cumulative percentage below } \\
\text { upper limit of class }\end{array}$ \\
\hline $\begin{array}{r}\text { up to }-3.0 \mathrm{sd} \text { incl. } \\
\text { over }-3.0 \text { sd to }-2.0 \text { sd incl. }\end{array}$ & 0.14 \\
over -2.0 sd to $-1.0 \mathrm{sd}$ incl. & 2.28 \\
over -1.0 sd to 0.0 sd incl. & 15.87 \\
over 0.0 sd to +1.0 sd incl. & 50.00 \\
over +1.0 sd to +2.0 sd incl. & 84.13 \\
over +2.0 sd to +3.0 sd incl. & 97.72 \\
over +3.0 sd & 99.87 \\
\hline
\end{tabular}

The table (Fisher, 1966) presents percentage areas for two to thirteen categories, but it is suggested that use of eight categories would present the greatest advantage because: (a) coding in this number of steps can be accommodated in one column of punched cards, still leaving the entries 0 or 9 free for "no answer," or unclassifiable responses; and (b) an even number of steps permits "median splits" which may be advantageous for analysis of subgroups.

For eight categories, dividing the range from -4.0 to +4.0 standard deviations into equal steps, percentage frequencies give the cumulative percentages shown in Table 1.

\begin{tabular}{|c|c|c|c|c|c|}
\hline Score & frequency & $\begin{array}{l}\text { cumulative } \\
\text { frequency }\end{array}$ & $\begin{array}{l}\text { percentage } \\
\text { cumulative } \\
\text { frequency }\end{array}$ & $\begin{array}{l}\text { cutting } \\
\text { points } \\
\text { (a) }\end{array}$ & $\begin{array}{l}\text { transformed } \\
\text { scores } \\
\text { (b) }\end{array}$ \\
\hline 0 & 0 & 0 & 0 & * & 1 \\
\hline 1 & 2 & 2 & 2.35 & * & 2 \\
\hline 2 & 3 & 5 & $5 . \overline{88}$ & & \\
\hline 3 & 6 & 11 & 12.94 & & 3 \\
\hline 4 & 3 & 14 & $16 . \underline{47}$ & $-*$ & \\
\hline 5 & 6 & 20 & 23.53 & & \\
\hline 6 & 3 & 23 & 27.06 & & \\
\hline 7 & 7 & 30 & 35.29 & & 4 \\
\hline 8 & 7 & 37 & 43.53 & & \\
\hline 9 & 6 & 43 & 50.59 & * & \\
\hline 10 & 5 & 48 & 56.47 & & \\
\hline 11 & 4 & 52 & 61.18 & & \\
\hline 12 & 1 & 53 & 62.35 & & \\
\hline 13 & 1 & 54 & 63.53 & & \\
\hline 14 & 3 & 57 & 67.06 & & \\
\hline 15 & 3 & 60 & 70.59 & & \\
\hline 16 & 2 & 62 & 72.94 & & 5 \\
\hline 17 & 1 & 63 & 74.12 & & \\
\hline 18 & 1 & 64 & 75.29 & & \\
\hline 19 & 4 & 68 & 80.00 & & \\
\hline 20 & 1 & 69 & 81.17 & & \\
\hline 21 & 0 & 69 & 81.17 & & \\
\hline 22 & 2 & 71 & 83.53 & & \\
\hline 23 & 0 & 71 & 83.53 & * & \\
\hline 24 & 3 & 74 & 87.06 & & \\
\hline 25 & 2 & 76 & 89.41 & & \\
\hline 26 & 2 & 78 & 91.76 & & \\
\hline 27 & 1 & 79 & 92.94 & & 6 \\
\hline 28 & 1 & 80 & 94.12 & & \\
\hline 29 & 2 & 82 & 96.47 & & \\
\hline 30 & 1 & 83 & 97.65 & & \\
\hline 31 & 0 & 83 & 97.65 & * & . \\
\hline 32 & 1 & 84 & 98.82 & & \\
\hline 33 & 0 & 84 & 98.82 & & \\
\hline 34 & 0 & 84 & 98.82 & & \\
\hline 35 & 0 & 84 & 98.82 & & 7 \\
\hline 36 & 0 & 84 & 98.82 & & \\
\hline 37 & 0 & 84 & 98.82 & & \\
\hline 38 & 0 & 84 & 98.82 & * & \\
\hline \multirow[t]{2}{*}{39} & 1 & 85 & 100.00 & & 8 \\
\hline & 85 & & & & \\
\hline
\end{tabular}


Table 3

Transformed Score

\begin{tabular}{lr}
1 & 0 \\
2 & 2 \\
3 & 12 \\
4 & 29 \\
5 & 28 \\
6 & 12 \\
7 & 1 \\
8 & 1 \\
\hline
\end{tabular}

An example taken from actual experimental data illustrates the use of the suggested technique (Table 2): (a) cutting points have been taken as closely as possible to theoretical limits, and (b) all scores falling between two cutting points have been allotted the transformed score as shown.

The frequency tabulation of scores after transformation is given in Table 3 and clearly indicates the close approximation to normal distribution.

References

FISHER, G. H. The New Form Statistical Tables (2nd Edition) London: University of London Press, 1966.

FISHER, G. H. Consideration of the normality of experimental data. Psychon. Sci, 1967, in press.

Note

1. The author is indebted to Dr. Gerald H. Fisher for encouragement in preparing this note for publication. 